\title{
Experimental evaluation of an open source implementation of IPv6 GeoNetworking in VANETs
}

\author{
Thouraya TOUKABRI*, Manabu TSUKADA* ${ }^{* \dagger}$, Thierry ERNST ${ }^{* \dagger}$ and Lamjed BETTAIEB ${ }^{\ddagger}$ \\ Email: \{thouraya.toukabri,manabu.tsukada and thierry.ernst \}@inria.fr, lamjed.bettaieb@esprit.ens.tn \\ * INRIA Rocquencourt - IMARA Project-Team \\ Domaine de Voluceau, BP 105 78153, Le Chesnay, FRANCE \\ ${ }^{\dagger}$ Mines ParisTech - CAOR Lab \\ 60, Boulevard Saint-Michel 75272 Paris, FRANCE \\ $\ddagger$ ESPRIT - Tunis \\ 18, rue de l'Usine - ZI Aéroport, Charguia II, 2035 Ariana, TUNISIA
}

\begin{abstract}
ISO TC204 and ETSI TC ITS are developing a set of standards for Cooperative ITS (Cooperative Intelligent transportation Systems) which will allow ITS stations i.e. vehicles, the road infrastructure and other peers reachable through the Internet to cooperate and exchange information with one another in order to enhance road safety, traffic efficiency and comfort for all road users. In situations where the exchange of information has to transit through the Internet, the use of IP, more specifically IPv6, is crucial and meets ITS needs for reliable and scalable communication capabilities in vehicular networks. An implementation of Cooperative ITS communication protocols is necessary to validate extensively the ETSI and ISO Cooperative ITS standards. In this paper, we describe CarGeo6, an ongoing open-source implementation of the IPv6 GeoNetworking capabilities of the ITS station reference architecture based on the output of the GeoNet European Project. CarGeo6 combines IPv6 and GeoNetworking capabilities into a common protocol stack for the transmission of IPv6 packets into a given geographical area. This paper reports the validation process and the network performance evaluation of CarGeo6 as well as a comparison of these results with GeoNet results.
\end{abstract}

\section{INTRODUCTION}

Road safety, fleet control, Geo-localization, infotainment and other applications are new road services that need not only new control technologies for vehicles and the infrastructure (sensors capacity, high image processing, complex data handling...) but also efficient data transmission between vehicles and the infrastructure. A new vision of Intelligent Transportation Systems (ITS) is born: Cooperative ITS. The idea is based on conceiving and deploying a transportation system in which the roadside infrastructure, vehicles, and remote entities cooperate to enhance road safety, traffic efficiency and comfort of road users (drivers, passengers, pedestrians, fret carriers). With this new revolutionary concept of Cooperative ITS, the pure fiction of communicating vehicles is becoming a reality in which suitable communication forms, architectures and techniques are deployed to combine in an efficient way the internal control function of the vehicle with its external communication function.

As we are talking about vehicles moving at high speed on highways, a geographic position-based routing protocol (GeoNetworking) was defined and specified by the Car to Car
Communication Consortium $(\mathrm{C} 2 \mathrm{C}-\mathrm{CC})^{1}$ in order to distribute information to all vehicles in a given road area. Moreover, in situations where the exchange of information has to transit through the Internet and considering today's Internet predomination in mostly all communication systems, Internet-based communication scenarios are crucial for vehicular communications in order to keep the whole system up to date with the new technological achievements in favor of the ubiquitous Internet. IPv6 takes its place within these new ITS communication architectures, thanks to its advantages like extended address space, embedded security, enhanced mobility support and ease of configuration, in boosting new Internet and ITS usages.

IPv6 is then an opportunity to use the Internet differently in ITS and expanding it with GeoNetworking could result in an efficient, scalable (addresses for an unlimited number of vehicles) and flexible (the protocol is extensible and any underlying wireless technology could be accommodated) vehicular communication system for safer roads and a sustainable development of the road network.

The GeoNet European Project ${ }^{2}$ was the first experimental work investigating the use of IPv6 in vehicular communications. The project ran for two years between 2008 and 2010 and was setup to study, implement and demonstrate the combination of IPv6 with GeoNetworking capabilities as designed by the $\mathrm{C} 2 \mathrm{C}-\mathrm{CC}$. The project issued an architecture and the functional specification of the architecture components [1], [2]. It provided two implementations of C2C-CC's GeoNetworking capabilities and their integration with an IPv6 stack running on top and extended with additional features. The project proved the concept combining both IPv6 and GeoNetworking. It also published experimental test results and a performance evaluation conducted both indoor and outdoor to evaluate the performance of IPv6 GeoNetworking [3], [4].

Unfortunately, both implementations of the GeoNetworking capabilities developed within the GeoNet project were proprietary. Only the binary code was made available to other GeoNet partners. Considering the lack of access to the code

\footnotetext{
${ }^{1}$ C2C-CC:www.car-to-car.org

${ }^{2}$ GeoNet project: www.geonet-project.eu
} 
as a restraint for the continuous validation and performance improvement of IPv6 GeoNetworking, the idea of developing an open source prototype of the IPv6 GeoNetworking protocol stack came. Indeed, an open source implementation is justified by the fact that open source has gained more ground with the rise of the Internet in the recent past years. The promise of open source is mainly of a better quality, higher reliability, more flexibility, and a lower cost. It will also provide a playground to all Cooperative ITS stakeholders who have no knowledge in networking but need the necessary communications capabilities to test new applications.

This paper introduces CarGeo6 ${ }^{3}$, an open source implementation of IPv6 GeoNetworking. The project is a Linux-based prototype implementation that has been developed conforming with GeoNet specifications [2]. CarGeo6 has been validated and tested in the indoor testbed and outdoor. Network performance has been evaluated in different scenarios that involved sometimes only vehicles (V2V: Vehicle-to-Vehicle), vehicles and the roadside infrastructure (V2R: Vehicle to Roadside) or vehicles and the Internet (V2I: Vehicle to Internet).

The paper is structured as follows: First, we introduce the general ITS station reference architecture the CarGeo6 basic concepts rely on. In the third and the fourth sections, we highlight CarGeo6 particularities and the enhancements it brought to IPv6 GeoNetworking and to the architecture design. Then, the last section is dedicated to the report of some preliminary experimental results on the network performance.

\section{THE ITS STATION REFERENCE ARCHITECTURE}

ISO TC204 WG16, known as CALM (Communications Access for Land Mobiles) is working for ten years on a communication architecture supporting a variety of media types (infrared, microwave, $2 \mathrm{G} / 3 \mathrm{G}, \ldots$ ), a variety of networking protocols (IPv6, FAST) for a variety of Cooperative ITS needs (communication profiles) and access network load. Such an interface management component requires input from various layers and is thus a cross-layer function. With such a cross layer function combined with IPv6 mobility support functions (NEMO, MCoA) [5], continuous Internet connectivity can be maintained over transient communication interfaces (i.e. not breaking ongoing communications after vertical handover from e.g. 802.11p to $3 \mathrm{G}$ ) or transient access points (from one roadside ITS station supporting $802.11 \mathrm{p}$ to another one). This architecture has been developed, validated and demonstrated in the CVIS project [6] for a number of Cooperative ITS applications.

In parallel, the $\mathrm{C} 2 \mathrm{C}-\mathrm{CC}$ worked on a communication architecture and the specification of the GeoNetworking protocol for multi-hop $802.11 \mathrm{p}$ communications between vehicles and the road infrastructure.

In an effort towards harmonization, the European Commission's COMeSafety Specific Support Action issued an European ITS Communication Architecture which has then led to the definition of a uniform communication architecture

\footnotetext{
${ }^{3}$ CarGeo6 open source implementation: www.cargeo6.org
}

known as the ITS station reference communication architecture (see Figure 1) standardized at both the European level within ETSI TC ITS (Technical Committee for Intelligent Transport Systems) and the international level within ISO TC 204 (WG16). Both ISO and ETSI architecture specifications [7], [8] are based on the same terminology and tend to converge although there are still differences between the two until all standards composing this architecture are revised and aligned.

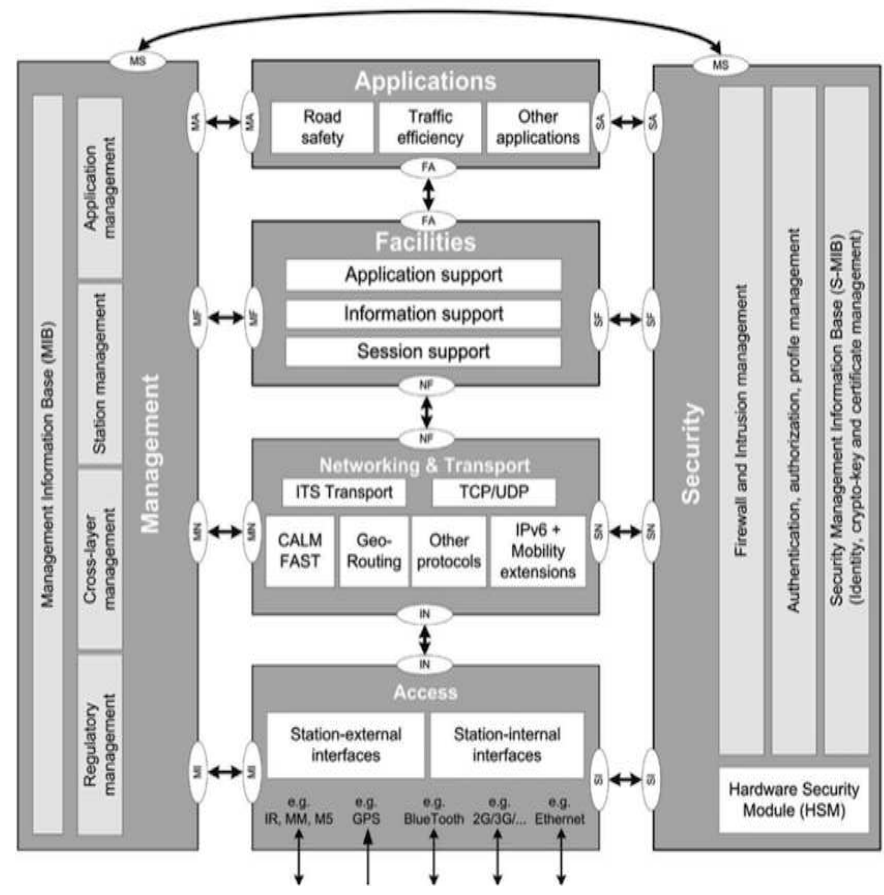

Fig. 1. ITS station reference architecture

The cross-layer design originally introduced by ISO TC204 WG16 is now clearly represented on the architecture diagram (ITS station management plane), though the cross-layer functions to be offered are still under discussion at the ETSI and ISO standardization level. Such an architecture would be deployed on various types of ITS stations involved in Cooperative ITS communications, but different features would be supported according to the type of ITS station, deployment environment and user needs. Types of ITS stations include the vehicle ITS station, the roadside ITS station, the personal ITS station and central ITS stations. The vehicle ITS station could be a private car, an emergency vehicle, a truck, a bus, a taxi, or even a tramway. The distinction is only made at the level of the subset of features and applications supported. The central ITS stations are typically road traffic servers, service providers, etc. The roadside ITS station type includes roadsigns, variable message signs (VMS), traffic lights, electric charging points and Internet access points dedicated for Cooperative ITS. The personal ITS station type includes nomadic devices such as mobile phones, digital car keys, etc. In the future, more ITS stations may be defined.

As a result, this ITS station architecture combines all types of communications: Vehicle-to-Vehicle (V2V) and Vehicle-to- 
Roadside (V2R) using IPv6 or GeoNetworking or a combination of both over IEEE 802.11p or other media; Vehicleto-Central (V2C) using IPv6 over any kind of media (e.g. $802.11 \mathrm{p}$ via a roadside ITS station, $802.11 \mathrm{n}$ via a public WIFI network, or $3 \mathrm{G}$ via the cellular network); Roadside-to-Central (R2C) using IPv6 over optic fiber, cellular networks, WiMaX. In this paper, we focus on $\mathrm{V} 2 \mathrm{~V}$ communications as described on Figure 2.

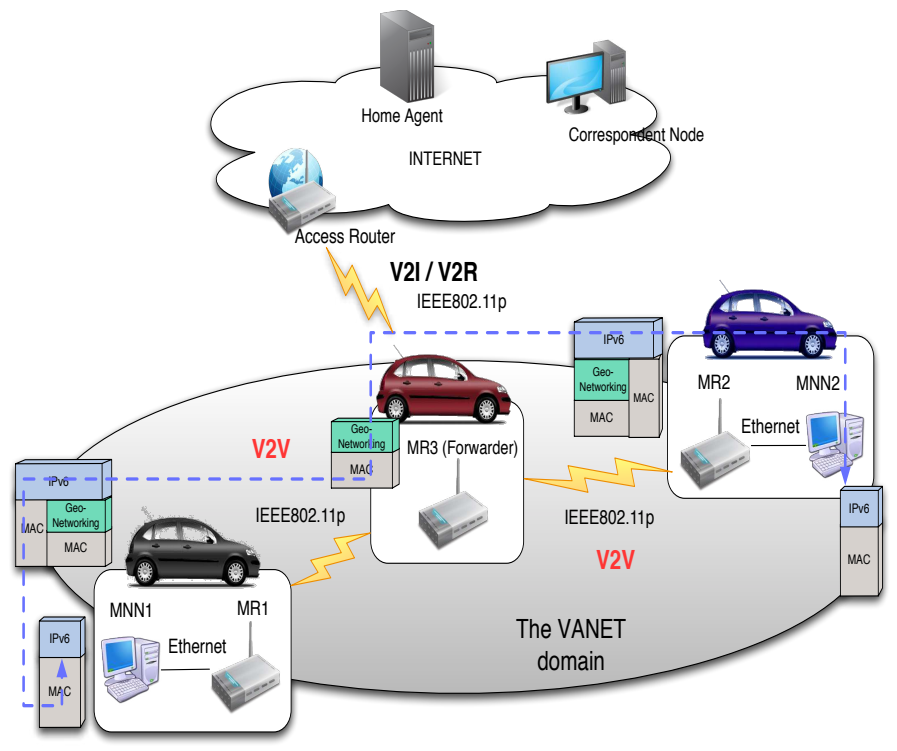

Fig. 2. V2V communications between ITS stations

\section{IPv6 GEONETWORKING ENHANCEMENTS}

The ITS station architecture illustrated in Figure 1 comprises multiple protocol stacks at the ITS station Network and Transport layer. As shown, GeoNetworking (GeoRouting on the figure) and IPv6 are two independent protocol stacks but these could be combined. The combination of IPv6 and GeoNetworking capabilities into a common protocol stack has been introduced and validated by the GeoNet project [1]. This resulted mainly in the design, integration and implementation of a set of components that allow sending IPv6 packets over a VANET managed by a GeoNetworking protocol. In addition to the GeoNetworking protocol (C2CNet), the newly developed components are mainly the adaptation of IPv6 over GeoNetworking (IPv6 over C2CNet) and Mobile Network Prefix Provisioning (MNPP ${ }^{4}$ ) [9]. In addition, some existing IPv6 features/components have also been integrated in the IPv6 stack such as MLDv2 to enable multicast over GeoBroadcast (Geographically scoped broadcast) and IPv6 mobility support (NEMO and MCoA) [10]. These contributions are detailed in the following sub-sections.

The transmission of IPv6 over GeoNetworking (i.e. IPv6 over $\mathrm{C} 2 \mathrm{CNet}$ ) has recently been specified and standardized

\footnotetext{
${ }^{4}$ MNPP: a solution to advertise the IPv6 prefix of a router (MRs and ARs) to other nearby routers in order to avoid the transmission of packets via the HA when routers are directly reachable over the IPv6 C2CNet link or any conventional wireless link.
}

by ETSI [11], based on GeoNet results, but this standard doesn't contain all the capabilities specified by the GeoNet project (it only allows to route IPv6 packets over a link with GeoNetworking capabilities, but not to geographically distribute IPv6 packets as realized by the GeoNet project).

Overall, the contributions of the GeoNet project proved to the ITS community that both IPv6 and GeoNetworking is needed for several ITS safety and traffic efficiency applications and proved the feasibility of this concept in real experiments reported in [3].

\section{A. C2CNet: a GeoNetworking protocol}

$\mathrm{C} 2 \mathrm{CNet}$ is a GeoNetworking protocol proposed by the $\mathrm{C} 2 \mathrm{C}$ $\mathrm{CC}$ and then implemented and experimented by the GeoNet project. $\mathrm{C} 2 \mathrm{CNet}$ is defined as a communication layer that enables GeoNetworking mechanisms (geographical addressing and routing). $\mathrm{C} 2 \mathrm{CNet}$ includes position-based routing mechanisms adapted to vehicular communications and use, in particular, the Greedy Perimeter Stateless Routing (GPSR) algorithm [12].

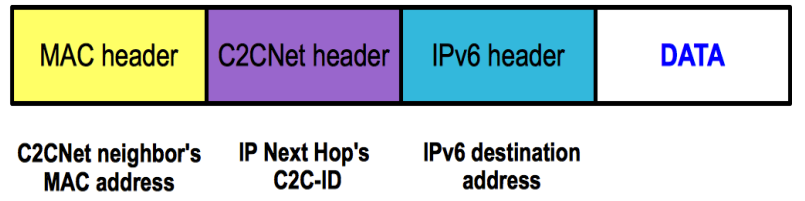

Fig. 3. IPv6 GeoNetworking packet headers

GPSR uses the greedy forwarding algorithm which routes data using information of direct neighbors (in the same wireless range). Besides, the $\mathrm{C} 2 \mathrm{CNet}$ mechanism defines a new network header that carries the $\mathrm{C} 2 \mathrm{C}$ identifier (identifier of the ITS station) of the source and the destination as well as their geographic positions acquired from the Positioning module (see Figure 3). Each node in the VANET domain is then identified by its unique C2CNet ID which is exchanged between the vehicle and its direct neighbors.

In addition, C2CNet defines also a mechanism for geographic multi-hop communications, in which a given vehicle could communicate with another vehicle not in his wireless range. This mechanism is called Location Service and provides a Request/Reply based mechanism that allows the source vehicle to request if the destination is in the wireless range of one of its immediate neighbors. In this case, intermediate nodes are called $\mathrm{C} 2 \mathrm{CNet}$ Forwarders and are in charge of relaying the message until the destination using $\mathrm{C} 2 \mathrm{CNet} \mathrm{Geo}-$ Routing mechanisms.

\section{B. Adaptation of IPv6 over C2CNet}

The IPv6 over C2CNet sub-module as specified in GeoNet [2] is the adaptation module that allows passing IPv6 packets coming from the IPv6 stack to the $\mathrm{C} 2 \mathrm{CNet}$ layer. This submodule is in charge of acquiring necessary IPv6 parameters such as the IPv6 address, performing IP Next Hop determination and IPv6 address resolution over the C2CNet link. IPv6 packets are then sent to $\mathrm{C} 2 \mathrm{CNet}$ through the GeoIP SAP 
(Service Access Point between IP and GeoNetworking), the piece of software that links the IPv6 layer to the $\mathrm{C} 2 \mathrm{CNet}$ layer. All incoming packets from the IPv6 stack and going to the wireless interface are mapped according to their packet type into $\mathrm{C} 2 \mathrm{CNet}$ packets with a given destination range. The mapping from IPv6 schemes to $\mathrm{C} 2 \mathrm{CNet}$ schemes is done as shown in table I.

TABLE I

SCHEMES MAPPING BETWEEN IPV6 AND C2CNET

\begin{tabular}{|l|l|l|}
\hline Destination range & IPv6 scheme & C2CNet scheme \\
\hline \hline A node in a specific vehicle & Unicast & GeoUnicast \\
\hline $\begin{array}{l}\text { Nodes located in vehicles in a specific } \\
\text { geographical area }\end{array}$ & Multicast & GeoBroadcast \\
\hline $\begin{array}{l}\text { A node located in a vehicle in a specific } \\
\text { geographical area }\end{array}$ & Anycast & GeoAnycast \\
\hline A node in vehicles at x hops away & Multicast & TopBroadcast \\
\hline \hline
\end{tabular}

Application data is encapsulated in IPv6, C2CNet and IEEE $802.11 \mathrm{p}$ [13] headers in that order and each header designates a different entity. At the $\mathrm{C} 2 \mathrm{CNet}$ layer, IPv6 packets are encapsulated with a $\mathrm{C} 2 \mathrm{CNet}$ header where the IP Next Hop is designated by the destination $\mathrm{C} 2 \mathrm{CNet}$ identifier. Until the packet reaches the IP Next Hop ${ }^{5}$, all intermediary nodes only check the packets $\mathrm{C} 2 \mathrm{CNet}$ header and ignore its IPv6 header. Only the IP Next Hop consults the IPv6 header to make a forwarding decision to the IPv6 destination node.

\section{Cross-Layer Functions}

In addition to the communication interface management function which is by design a cross-layer function, and as a result of the GeoNet project, INRIA is advocating for two additional cross-layer functions not yet part of the ITS station management plane: Positioning and Geo-destination. The Positioning module is in charge of computing a precise position of the vehicle based on the fusion of the information received by both the ITS station network and transport layer (GeoNetworking) and the ITS station facilities layer $\left(\mathrm{CAM}^{6}\right.$, $\mathrm{DENM}^{7}, \mathrm{LDM}^{8}$ ) [14]-[16]. The Geo-destination module is needed by the ITS station network and transport layer (IPv6 GeoNetworking) to determine in which geographical area an IPv6 multicast packet should be transmitted by the GeoNetworking sub-layer.

\section{IPv6 Multicast and GeoDestination}

According to GeoNet reference scenarios [1], many use cases of ITS road safety and traffic efficiency applications need to send a message to a set of nodes in the VANET within a specific geographical area. Existing solutions, such as unicasting the packets to those multiple destinations or broadcasting them in the entire VANET, do not provide the suitable mechanisms to ensure distributing packets in an efficient way from the

\footnotetext{
${ }^{5}$ Designates the next hop from an IP viewpoint. It is the destination node to which the packet is delivered by the $\mathrm{C} 2 \mathrm{CNet}$ forwarding mechanism

${ }^{6} \mathrm{CAM}$ : Cooperative Awareness Message

${ }^{7}$ DENM: Decentralized Environmental Notification Message

${ }^{8}$ LDM: Local Dynamic Map
}

sender to their receivers. The transmission of a packet to the set of interested nodes is known as multicasting, by opposition with broadcasting which is flooding all nodes.

The challenges in GeoNet were to extend IPv6 multicast so that only nodes within a certain geographic area could be addressed, i.e. geographically scoped multicast. Several approaches were discussed in [2] such as encoding the geographic coordinates of the geographic destination area (GeoDestination) in the IPv6 multicast address (which means providing a new IPv6 address format), recording the GeoDestination in a new extended header (which means providing extensions to the IPv6 protocol) or using encapsulation. The solution finally adopted in GeoNet is a solution making use of the cross-layer nature of the ITS station: the IPv6 multicast address is statically mapped to a corresponding GeoDestination ID which is in turn mapped to a GeoDestination at the ITS station management layer (Geo-destination module). The GeoDestination is provided to the $\mathrm{C} 2 \mathrm{CNet}$ module through the MN-SAP based on the GeoDestination ID embedded in the IPv6 multicast address and passed by IPv6 to C2CNet through the GeoIP SAP. IPv6 multicast packets are then simply GeoBroadcasted at the $\mathrm{C} 2 \mathrm{CNet}$ layer within the GeoDestination around the originating node or a target node. Nevertheless, GeoDestination is a new concept proposed in GeoNet but not entirely specified. It is still not being considered in ETSI and ISO standards.

\section{The CARGEO6 Open Source IMPLEMENTATION}

Issued from the collaboration between the Tunisian school ESPRIT and INRIA, the French institute of research in computer science, CarGeo6 is a stable open source implementation combining IPv6 and GeoNetworking protocol blocks of the ITS station network and transport layer of the reference architecture. The software is a Linux-based prototype programmed in the $\mathrm{C}$ language and released under a LGPLv2 license (Lesser GNU Public License).

The implementation currently comply with the architecture and specifications published by the GeoNet European Project [1], [2]. It ill be improved in the short term in order to comply with the most recent ETSI standards on GeoNetworking and IPv6 GeoNetworking [11], [17]. The prototype is validated on the same testing platform as the one used in the GeoNet project experimentations [3].

Figure 4 shows the implementation design of CarGeo6 and the origin of the contributions (from CarGeo6 partners or earlier sources). It is also envisioned to extend it with ITS station facilities layer capabilities (CAM, DENM, SAM ${ }^{9}$ and LDM) and ITS station management plane capabilities (interface management, remote ITS station management).

The GeoNetworking layer referred to as the C2CNet layer in the GeoNet documents and the SAPs between the ITS station Network and Transport layer and the ITS station access layer (IN-SAP) were implemented by ESPRIT. ESPRIT also provided an implementation of the Positioning module that

\footnotetext{
${ }^{9}$ SAM: Service Advertisement Message
} 


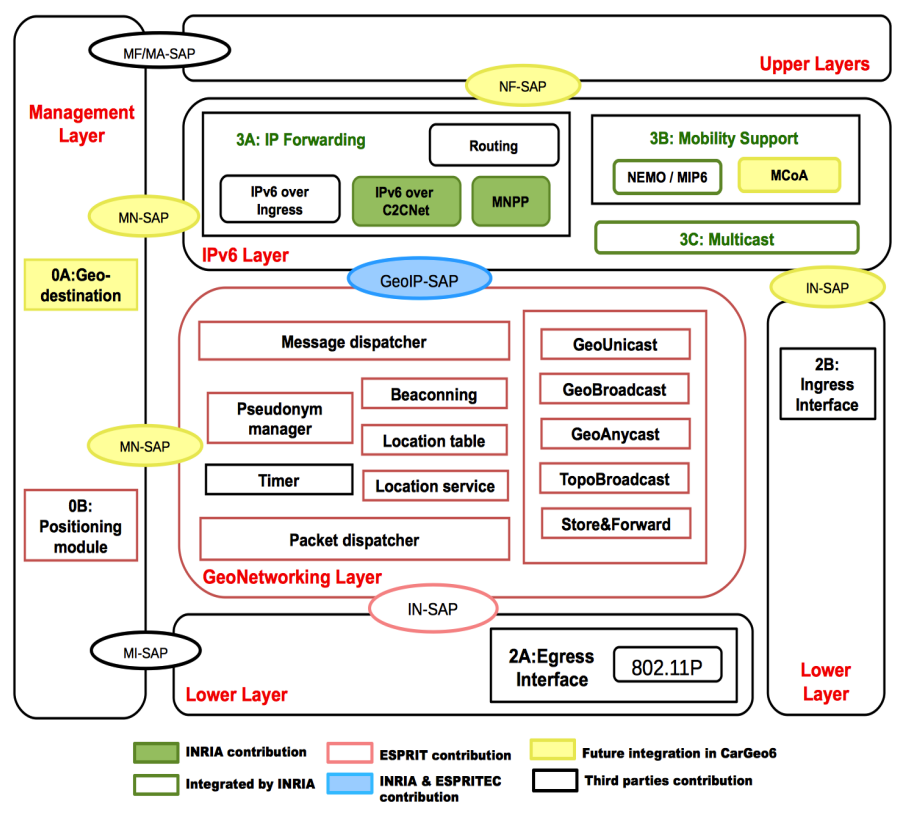

Fig. 4. CarGeo6 implementation design and contributions

sends local position information read from a GPS receiver to the GeoNetworking layer. This module will be extended in the future to support different positioning systems and combine their capabilities for a more precise positioning information in the vehicle.

INRIA contributed a protocol allowing direct communication between nearby ITS stations (Mobile Network Prefix Provisioning) [9], its integration with IPv6 mobility support (NEMO and MCoA) [10], IPv6 multicast (MLDv2) and the adaptation module between the IPv6 protocol suite and GeoNetworking (IPv6 over C2CNet). In particular, this latest component contains the internal GeoIP SAP that encapsulates IPv6 packets into GeoNetworking packets through a virtual TUN/TAP network interface.

The IPv6 stack being implemented in the kernel space, an adaptation module has been implemented in the user space in order to send IPv6 packets to the new GeoNetworking software implemented by ESPRIT in the user space. This adaptation module, referred as the IPv6 over C2CNet submodule in GeoNet documents, should perform the IP Next Hop determination: The software looks up in the main routing table using LibNetlink ${ }^{10}$ messages to find the IPv6 destination address and then brings up the information to the user land. At this level, the IPv6 destination address is matched with a unique destination GeoNetworking Identifier, referred as C2CNet ID in GeoNet. So far, the software performs a look up in the Location Table ${ }^{11}$ to determine if the destination could be reached using single hop Geo-Routing protocol (the IP Next Hop is a neighbor) or multi-hop Geo-Routing protocol.

Once the destination scope is determined, the GeoIP SAP

\footnotetext{
${ }^{10}$ LibNetlink: A library for accessing the netlink service in the linux kernel

${ }^{11}$ Location Table: a routing table implemented at the GeoNetworking layer that contains the list of neighbor vehicles and their C2CNet IDs
}

(see Figure 4), referred to as the C2C-IP SAP in GeoNet documents, creates and mounts a virtual TUN/TAP interface named tun 0 , performs the appropriate mapping operation on the IPv6 packet to a GeoNetworking packet and then sends it to the GeoNetworking layer. At this level, the appropriate header is added and the packet is send on the wireless link. The implementation design of the IPv6 GeoNetworking mechanism is illustrated in Figure 5 in the case of a multi-hop scenario.

CarGeo6 also implements IPv6 multicast over GeoNetworking as specified in GeoNet [1], [2]: IPv6 multicast packets are mapped at the GeoIP SAP into GeoBroadcast packets within a specific GeoDestination. The multicast group is then mapped tp a GeoDestination ID and a specific radius around a point given by its coordinates. CarGeo6 currently implements this mechanism with a static GeoDestination table maintained at the GeoNetworking layer inside the adaptation module. A future contribution from CarGeo6 will be to specify the GeoDestination module as a part of the ITS station management plane and ensure the communication between this module and the GeoNetworking layer through cross layer management functions (MN-SAP primitives).

\section{INDOOR PERFORMANCE EVALUATION OF CARGEO6}

In this section, we report experimental performance results of our CarGeo6 implementation used in V2V communication scenarios. Experiments were conducted in the indoor platform using single hop and multi-hop configurations at the GeoNetworking layer. We used the IEEE 802.11g wireless communication protocol for the ad-hoc network between MRs and Ethernet links between MRs and their Mobile Network Nodes (MNNs). MRs have both IPv6 and GeoNetworking capabilities, whereas MNNs are simple IPv6 hosts with no GeoNetworking capabilities. We only report end-to-end IPv6 unicast communications from in-vehicle host MNN1 attached to mobile router MR1 to in-vehicle host MNN2 attached to mobile router MR2, over the underlying GeoNetworking layer.

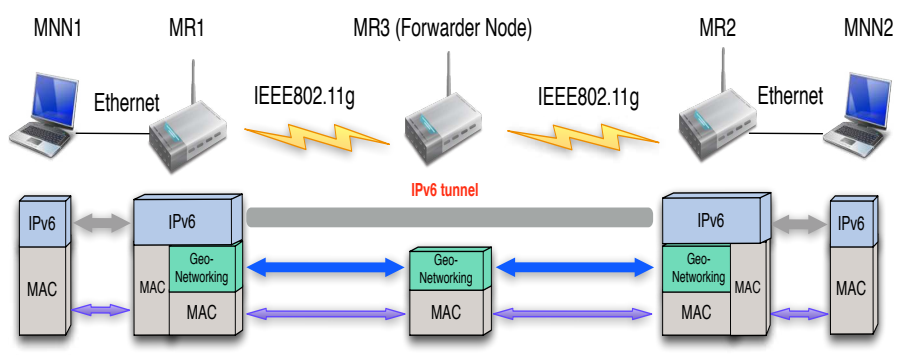

Fig. 6. Indoor testbed for V2V communications

Generally, the performance depends on the network configuration. In our case, it depends particularly on the number of GeoNetworking hops (C2CNet hops) over which packets are transmitted through from the source node (MNN1) to the destination (MNN2) and the overhead brought by the additional GeoNetworking header. As such, we considered both single hop and multi-hop configurations at the GeoNetworking level. 


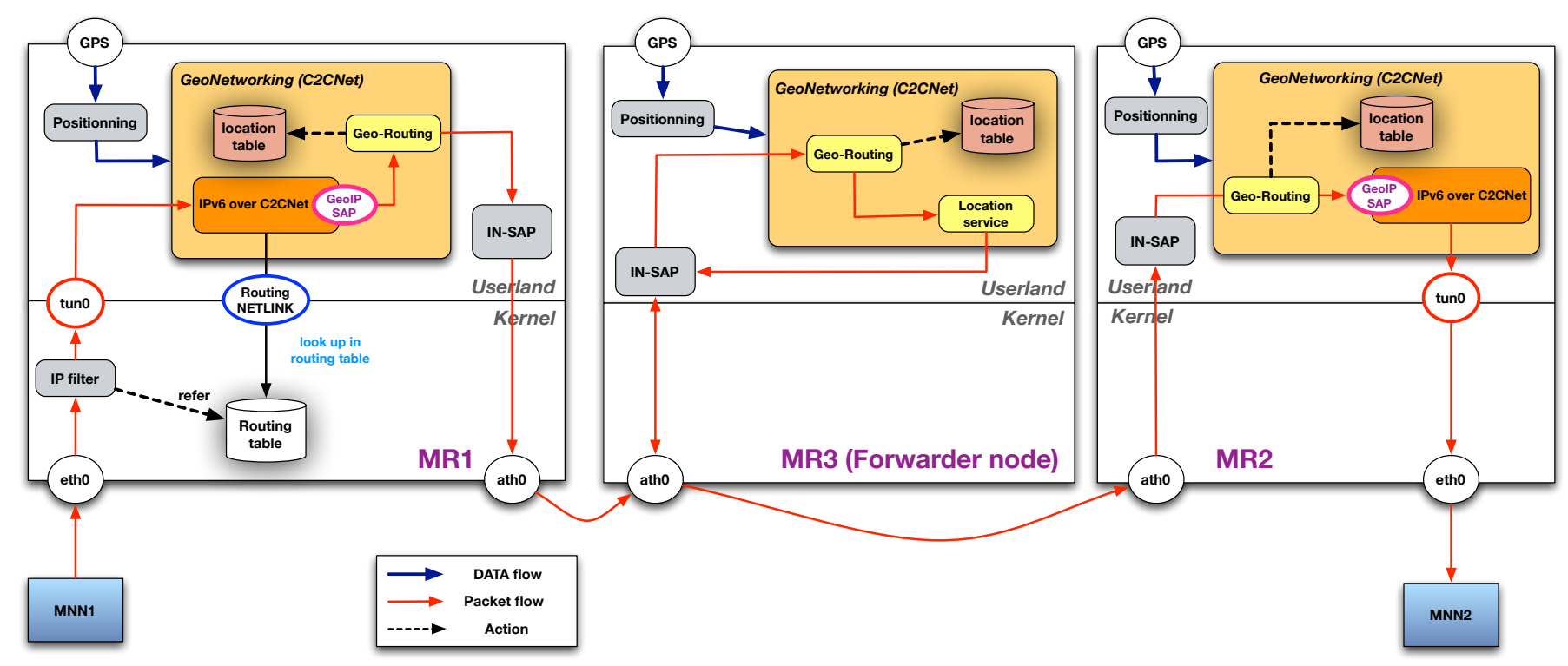

Fig. 5. Implementation of IPv6 over $\mathrm{C} 2 \mathrm{CNet}$ in CarGeo6

The testbed we set in our indoor platform (see Figure 6) is composed of 3 MRs and 2 MNNs. The test consists on sending IPv6 unicast packets from MNN1 to MNN2 using for the first experiment a direct link communication between MR1 and MR2 and in the second experiment using an intermediate GeoNetworking Node. As we could not use real GPS devices indoor due to poor signal reception, geographic positions of nodes are given by a static GPS sender software that runs in each of them.

\section{A. Evaluation methodology and metrics}

Our test methodology consists on executing scripts on the IPv6 hosts attached to each MR in order to evaluate several performance parameters. Results of the performance scripts are recorded in log files in IPv6 hosts and are parsed in order to generate graphs for each evaluation metric. The performance is evaluated according to some network metrics and configurable parameters summarized in Table II.

TABLE II

PARAMETERS AND METRICS FOR INDOOR PLATFORM PERFORMANCE EVALUATION

\begin{tabular}{|c|l|c|}
\hline \hline Flow type & Configurable parameters & Evaluation metrics \\
\hline \hline ICMPv6 & Packet size, sending interval, & RTT and packet loss \\
\hline \hline UDP & Buffer size, sending rate & Packet delivery ratio \\
\hline \hline TCP & $\begin{array}{l}\text { TCP window size, Maximum } \\
\text { segment size }\end{array}$ & Throughput \\
\hline
\end{tabular}

We note that the hop count is an important configurable parameter to take into consideration when evaluating the network performance of IPv6 GeoNetworking particularly the latency. Theoretically, we can get most basic network performance in a single hop configuration. On the other hand, the multihop configuration adds processing delay and overhead. In the following results, we experimented a multi-hop scenario with one hop forwarder.

To evaluate latency, we used ping6 to send ICMPv6 packets from MNN1 to MNN2 in order to measure the Round Trip Time (RTT) between the two end nodes. The purpose of using ping6 is to test Latency in bidirectional communications and the packet loss. Besides, we used iperf for the UDP performance evaluation: iperf is a client/server software that generates UDP traffic at the client side and collects information about the packet delivery ratio at the server side. Throughput is then calculated according to the data collected at the client side.

\section{B. Latency evaluation}

The latency is evaluated by the RTT value indicated in the ping 6 output. The ping 6 output indicates the minimum, maximum and average RTT for a given size of packet. The test consists on sending 100 ICMPv6 requests every $0.1 \mathrm{~ms}$ with different packet size values increased each time by 20 Bytes and varying from 20Bytes to 1500 Bytes. The ping6 output indicates also the packet loss average for each size of ICMPv6 packet. We report in Figure 7 and Figure 8 the results we had for a ping6 from MNN1 to MNN2 in both single hop and multi-hop configurations.

1) Single hop scenario: Figure 7 indicates first that there is packet loss for a packet size exceeding 1300Bytes. This is explained by the fact that we fixed the MTU of the TUN/TAP virtual interface to 1350Bytes in our test, which means that packets from 1320Bytes and more are automatically dropped as no fragmentation mechanism is neither enabled nor implemented at the TUN/TAP interface. The lack of a fragmentation mechanism at the GeoNetworking layer could also have an impact on the packet loss: The maximum MTU is fixed to 1500Bytes. The figure shows also that the average RTT for 
all packet size values varies mostly between $2 \mathrm{~ms}$ and $10 \mathrm{~ms}$ except for a packet size of 370 Bytes where we noticed a $25 \mathrm{~ms}$ maximum average RTT value with $8 \%$ packet loss.

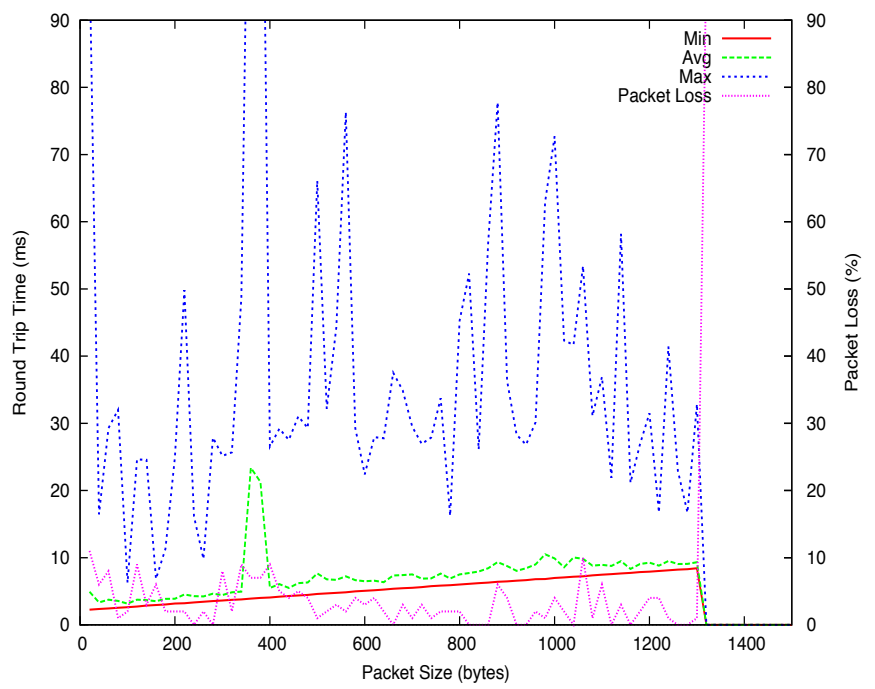

Fig. 7. ICMPv6 performance in single hop case

If we compare these results to GeoNet results [3], [4] for the same test, we can say that CarGeo6 average RTT values are globally better than GeoNet average RTT. Same as for the packet loss, values are almost similar in both implementations. However, these results could be improved by the implementation of an IP Next Hop cache. Currently, the IP Next Hop is resolved for each IPv6 packet at the IPv6 over C2CNet sub-module (adaptation module) which implies a processing delay on the RTT. The IP Next Hop cache avoids the software resolving the IP Next Hop address for packets having the same destination address. In other words, the cache will keep in a periodically refreshed table the destination C2CNet ID of an IP Next Hop and will not repeat this operation for packets having the same destination.

Besides, the packet loss values (maximum of $11 \%$ ) could also be improved. Even if the indoor testbed is intended to minimize interferences impact on the experiment, we cannot suppress definitely this constrain that could be caused by wireless engines located in the proximity of the testbed. Thus, interference impact could be avoided by the choice of a less noisy wireless channel and the isolation of the testbed as well as possible. The activation of QoS at the wireless interface could also improve the packet loss but may imply unfortunately an overhead.

2) Multi-hop scenario: As depicted in Figure 8, we can see that global values of RTT and packet loss are significantly higher with one $\mathrm{C} 2 \mathrm{CNet}$ Forwarder node than in the single hop configuration. The minimum packet loss value is $40 \%$ for a 1340Bytes packet size. Moreover, as in single hop case, packets are lost for packet size values over 1350Bytes due to the lack of fragmentation mechanisms at the $\mathrm{C} 2 \mathrm{CNet}$ layer. The maximum average RTT is also noticed for a 370Bytes packet size. Globally, RTT values in multi-hop are about 10 times higher than RTT values in single hop case and more than $40 \%$ packets are lost.

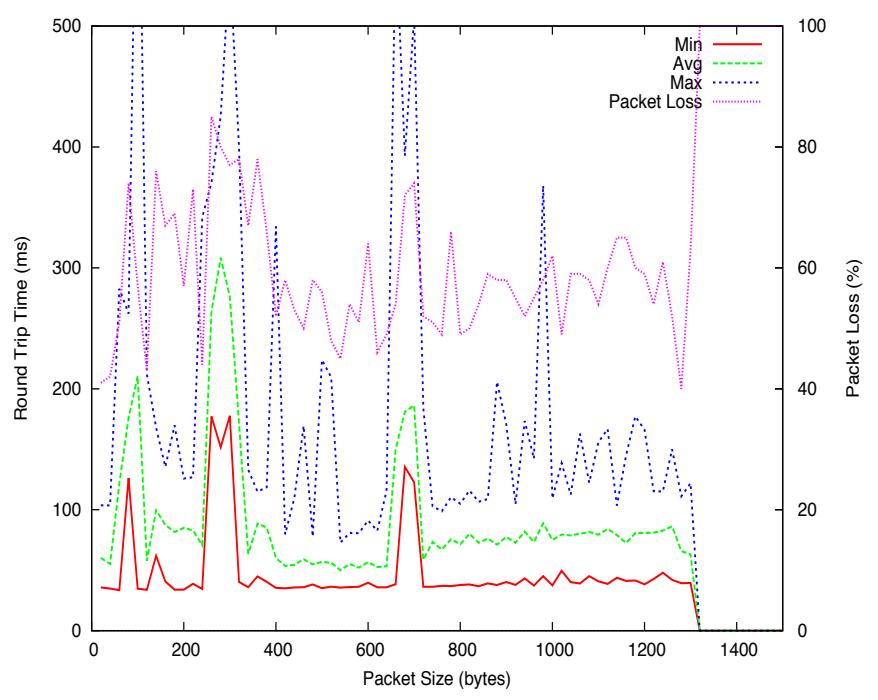

Fig. 8. ICMPv6 performance in multi-hop case

As written before, RTT values could generally be improved by the implementation of an IP Next Hop cache but we assume that this is not sufficient in the multi-hop case. RTT and packet loss high values are caused also by the Location Service mechanism implemented at the $\mathrm{C} 2 \mathrm{CNet}$ level. This mechanism is responsible for finding the $\mathrm{C} 2 \mathrm{CNet}$ ID of a node not in the neighborhood of the source. A process of Request/Reply packets is then triggered in order to find that ID. This mechanism implies a lot of waiting time until the source gets the reply with the C2CNet ID of the destination: the more we have intermediary nodes the bigger is the RTT and chances of packet loss. To improve this, a multi-hop beaconing mechanism where the source beacon is relayed until the destination through intermediary $\mathrm{C} 2 \mathrm{CNet}$ forwarders could be added.

\section{Overhead between GeoNetworking and IPv6}

In order to evaluate the overhead between IPv6 and GeoNetworking, we compare in Figure 9 the RTT values for different packet size for IPv6 without GeoNetworking and for IPv6 with GeoNetworking. The figure shows that the overhead between IPv6 and GeoNetworking in the single hop case is about 3ms, while it reaches $30 \mathrm{~ms}$ in the multi-hop case. We think that this overhead (multi-hop case) could be reduced if we implement the multi-hop beaconing mechanism instead of the Location Service mechanism.

\section{UDP performance in Single hop scenario}

In this part, we report UDP performance results for the single hop case. The performance is evaluated according to packet delivery ratio values and the throughput at the receiver side. The test consists on varying the datagram size from 100Bytes to 1900Bytes for different values of the UDP sending rate varying from $250 \mathrm{Kbits} / \mathrm{sec}$ to $2 \mathrm{Mbits} / \mathrm{sec}$. 


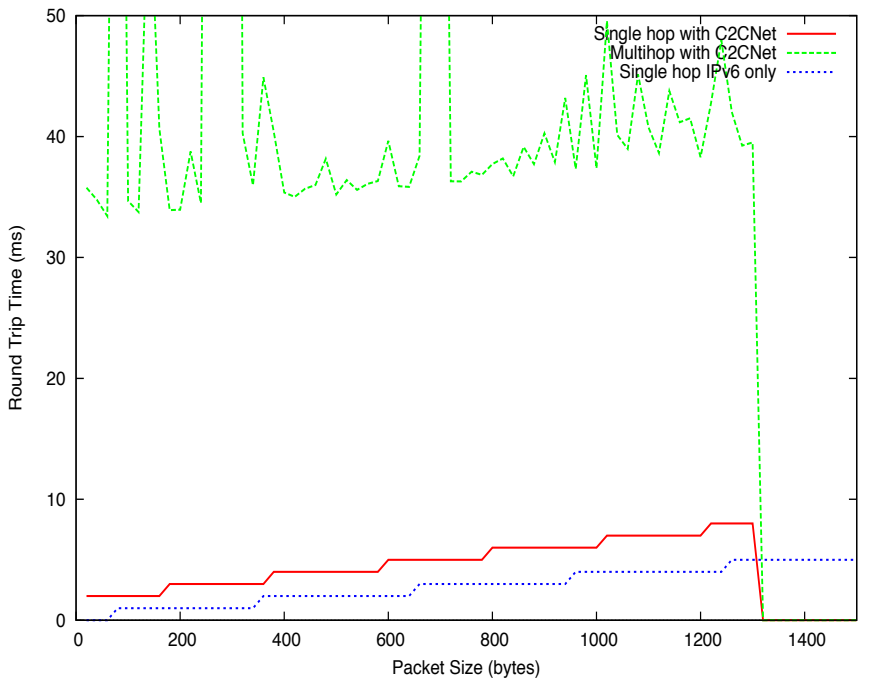

Fig. 9. Overhead between C2CNet and IPv6

Figure 10 shows the packet delivery ratio in the single hop case. The packet delivery ratio is low when the datagram size is too small: $60 \%$ packets are delivered for a 700Bytes datagram size and $250 \mathrm{Kbits} / \mathrm{sec}$ sending rate. The maximum packet delivery values (97\% to $100 \%$ ) are registered for a datagram size between 1150Bytes and 1380Bytes and with $250 \mathrm{Kbits} / \mathrm{sec}$ sending rate. Though, only $50 \%$ packet delivery is registered for these same datagram sizes with $1 \mathrm{Mbits} / \mathrm{sec}$ sending rate.

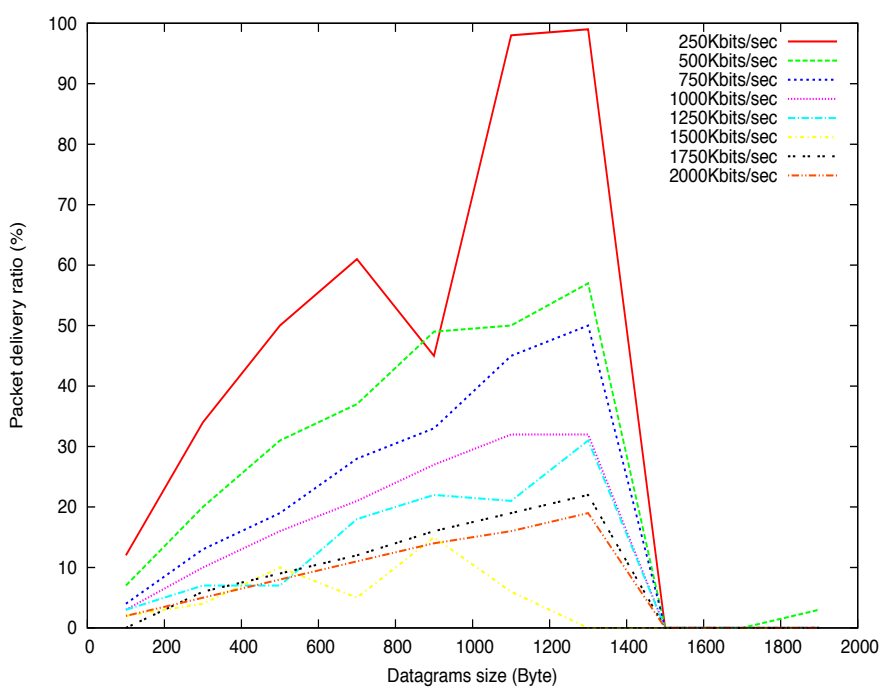

Fig. 10. UDP performance in single hop case

Figure 11 shows the throughput delivered in the same test. Throughput is maximized for all rates with 1 360Bytes datagram size. Besides, the maximum throughput value is registered for 420Bytes datagram size with $1250 \mathrm{Kbits} / \mathrm{sec}$, $1750 \mathrm{Kbits} / \mathrm{sec}$ and $2 \mathrm{Mbits} / \mathrm{sec}$ sending rates. We decided to limit our measurement interval to $2 \mathrm{Mbits} / \mathrm{sec}$ sending rate because packets are dropped for rates more than this value.
In comparison with GeoNet results for UDP performance [3], [4], CarGeo6's performance for UDP is currently poor but could be improved. Possible reasons for this results could be first wireless media issues due to interference as mentioned before. The same test will be done in the future with the $802.11 \mathrm{p}$ wireless media.

Besides, the quality of the $\mathrm{C} 2 \mathrm{CNet}$ link could also be the issue. As we suspect processing delays at the GeoNetworking layer, this could have an impact on the UDP traffic transmission from the source to the destination. To verify this assumption, we plan to develop a ping6-alike tool that measures the latency between two nodes at the GeoNetworking layer.

Currently, our assumption is the following: With the iperf tool, the server sends statistic information about the link state to the client (sender node) periodically after receiving a certain number of datagrams. If packets take too much time to arrive, and as UDP is an unreliable protocol, the server could send state information of the link before receiving the packets. This means that late arrived packets could be considered as lost. Moreover, we noticed according to the Figure that, the bigger the sending rate is, the lesser packets arrive to the destination. This could confirm the assumption that the processing time implies too much delay on the communication: the bigger the packet is, the bigger the processing delay will be, and the bigger the chance to lost the packet is.

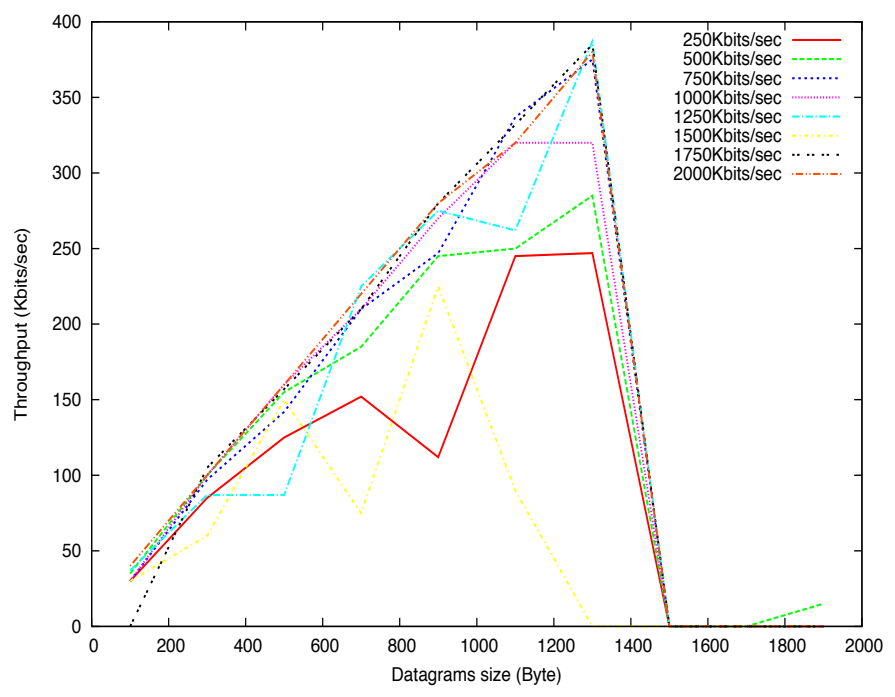

Fig. 11. UDP throughput in single hop case

\section{CONCLUSION AND NEXT STEPS}

In this paper, we presented CarGeo6, an open source implementation of IPv6 GeoNetworking complying to the GeoNet specifications [2]. We reported our first experimentation performance results of CarGeo6 for a single hop and multi-hop scenarios. We tried to evaluate some performance aspects of the implementation such as the latency, the packet delivery ratio for UDP and the UDP throughput. The current results analysis showed some performance issues. The performance 
could be enhanced with the implementation of some extension features like the IP Next Hop cache to enhance latency and multi-hop beaconing to enhance performance in the multi-hop case. We also plan to conduct more experiments in order to investigate the reasons of the poor performance in UDP and also TCP and bring out the current implementation to a more stable state.

Future work on CarGeo6 consists mainly on enhancing the indoor performance and testing the implementation outdoor using vehicles in more realistic scenarios. For this work, we took the GeoNet experiments as a reference [3]. Next, IPv6 over GeoNetworking will be adapted in order to comply with ETSI specifications on IPv6 GeoNetworking [11], [17].

Then, a second step is the integration in CarGeo6 of other components defined in the ITS station reference architecture, in particular ITS station facilities such as CAM (Cooperative Awareness Message), DENM (Decentralized Environmental Notification Message, SAM (Service Advertisement Message) and LDM (Local Dynamic Map) and cross-layer ITS station management capabilities such as interface management and selection. One of the key elements on which we are working also is the specification and definition of the SAPs between the Network and Transport layer and the other ITS station layers i.e the ITS station facilities and access layers and the ITS station security and management vertical planes.

\section{ACKNOWLEDGMENT}

The authors would like to thank the Tunisian developers from the ESPRIT school for their efforts in the development of the CarGeo6 GeoNetworking layer and for their collaboration with the IMARA team (INRIA) for bringing CarGeo6 to its present state. Special thanks goes also to all IMARA team members for their support and help during CarGeo6 experimentations.

\section{REFERENCES}

[1] GeoNet Project. GeoNet STREP N. 216269 D1.2 Final GeoNet Architecture Design. 2010. GeoNet-D.1.2-v1.1.

[2] GeoNet Project. GeoNet STREP No. 216269 D2.2 Final GeoNet Specification. 2010. GeoNet-D.2.2-v1.1.

[3] GeoNet Project. GeoNet STREP No.216269 D7.1 GeoNet Experimentation Results. 2010. GeoNet-D7.1-ExperimentationResults-v1.0.

[4] Manabu Tsukada, Ines Ben Jemaa, Hamid Menouar, Wenhui Zhang, Maria Goleva, and Thierry Ernst. Experimental evaluation for IPv6 over VANET geographic routing. In IWCMC '10: Proceedings of the 6th International Wireless Communications and Mobile Computing Conference, pages 736-741, New York, NY, USA, 2010. ACM.

[5] ISO/DIS 21210.2 Intelligent transport systems - Communications access for land mobiles (CALM) - IPv6 Networking, January 2011. ISO 21210:2011(E).

[6] Marie Moe, Vilmos Nebehaj, and Thierry Ernst. CVIS Performance Test Results: Fast Handovers in an 802.11p Network. In ITST '10: Proceedings of the 10th International Conference on Telecommunications for Intelligent Transport Systems, Kyoto, Japan, 2010.

[7] Intelligent Transport Systems (ITS); Communications Architecture, September 2010. ETSI EN 302665 V1.1.1 (2010-09).

[8] ISO 21217:2010 Intelligent transport systems - Communications access for land mobiles (CALM) - Architecture, April 2010.

[9] Jong-Hyouk Lee, Manabu Tsukada, and Thierry Ernst. Mobile network prefix provisioning (mnpp), October 2009. IETF, Work in progress, draft-jhlee-mext-mnpp-00.
[10] V. Devarapalli, R. Wakikawa, A. Petrescu, and P. Thubert. Network Mobility (NEMO) Basic Support Protocol. RFC 3963 (Proposed Standard), January 2005.

[11] Intelligent Transport Systems (ITS); Vehicular Communications; GeoNetworking; Part 6: Internet Integration; Sub-part 1: Transmission of IPv6 Packets over GeoNetworking Protocols, March 2011. ETSI TS 102 636-6-1 V1.1.1 (2011-03).

[12] Brad Karp and H. T. Kung. Gpsr: Greedy perimeter stateless routing for wireless networks. In 6th Annual International Conference on Mobile Computing and Networking, MobiCom 2000, August 6.-11., 2000, Boston, Massachusetts, USA, pages 243-254. ACM / IEEE, August 2000.

[13] IEEE Standard for Information technology - Telecommunications and information exchange between systems - Local and metropolitan area networks - Specific requirement, Part 11: Wireless LAN Medium Access Control (MAC) and Physical Layer (PHY) Specifications, July 2010. IEEE Std $802.11 \mathrm{p}-2010$.

[14] Intelligent Transport Systems (ITS); Vehicular Communications; Basic Set of Applications; Part 2: Specification of Cooperative Awareness Basic Service, March 2011. ETSI TS 102 637-2 V1.2.1 (2011-03).

[15] Intelligent Transport Systems (ITS); Vehicular Communications; Basic Set of Applications; Part 3: Specifications of Decentralized Environmental Notification (DENM) Basic Servic, September 2010. ETSI TS 102 637-3 V1.1.1 (2010-09).

[16] Intelligent transport systems (its); vehicular communications; basic set of applications; local dynamic map (ldm); rationale for and guidance on standardization, June 2011. ETSI TR 102863 V1.1.1 (2011-06).

[17] Intelligent Transportation Systems (ITS); Transport \& Network; Vehicular Communications; Part 1: Requirements for GeoNetworking and Data Transport Protocol, March 2010. ETSI TS 102 636-1 V1.1.1 (2010-03). 